\title{
Synthesis and Electrochemical Performance of a PEDOT:PSS@Ge Composite as the Anode Materials for Lithium-Ion Batteries
}

\author{
Jinfeng Liu, Jing Xu*, Yufang Chen, Weiwei Sun, Xiaoxiong Zhou, Jianhuang Ke \\ college of Aerospace Science and Engineering, National University of Defense Technology, Chang \\ Sha, Hu Nan, 410073, China \\ *E-mail: xujin503@163.com
}

doi: $10.20964 / 2019.01 .08$

Received: 18 September 2018 / Accepted: 16 October 2018 / Published: 30 November 2018

\begin{abstract}
The theoretical specific capacity and volume specific capacity of Ge anode active materials can reach to $1600 \mathrm{mAhg}^{-1}$ and $8500 \mathrm{mAh} / \mathrm{cm}^{3}$, respectively, which offers a significant advantage to high-power lithium-ion batteries used for miniaturization and weight reduction. However, the volume expansion rate of $\mathrm{Ge}$ is as high as $300 \%$ during cycling, resulting in poor first Coulomb efficiencies and cyclic stabilites. In this paper, a new type of organic shell (PEDOT: PSS) @ inorganic core (Ge) composite anode material for lithium ion batteries was designed and synthesized by a simple solution impregnation method. The nano-Ge anode active material was prepared by a simple liquid phase reduction method. The composition, structure and electrochemical properties of the prepared composites were analysed by field emission scanning electron microscopy (SEM), transmission electron microscopy (TEM), X-ray diffraction (XRD), energy spectrum analysis (EDS), infrared spectroscopy (FT-IR), galvanostatic charge-discharge and alternating current impedance. The results show that the conductive polymer, PEDOT:PSS, was successfully coated onto the surface of the prepared nano-Ge particles. The composite electrodes exhibits a reversible capacity of $405 \mathrm{mAhg}^{-1}$ after 200 cycles at $0.2 \mathrm{C}$ and rate capability of 800 and $700 \mathrm{mAhg}^{-1}$ at $2 \mathrm{C}$ and $4 \mathrm{C}$ respectively, which is much better than nanostructured Ge anodes without PEDOT:PSS coating. Meanwhile, there are higher initial discharge capacity (Up to $1400 \mathrm{mAhg}^{-}$ ${ }^{1}$ ) and Coulomb efficiencies (89\%, pure Ge is 81\%) for PEDOT@ Ge. The significantly enhanced cycle performance is attributed to the face that the PEDOT:PSS coating can effectively improve the electronic conductivity of Ge and provide buffer framework to reduce the volume change in electrochemical lithium reactions.
\end{abstract}

Keywords: PEDOT:PSS, Ge, conductive polymers, lithium-ion battery

\section{$\underline{\text { FULL TEXT }}$}


(C) 2019 The Authors. Published by ESG (www.electrochemsci.org). This article is an open access article distributed under the terms and conditions of the Creative Commons Attribution license (http://creativecommons.org/licenses/by/4.0/). 